\title{
HIGH-ASPECT-RATIO ROTARY POLYGON MICROMOTOR SCANNERS
}

\author{
A. A. Yasseen, J. N. Mitchell, D. A. Smith, and M. Mehregany \\ Microfabrication Laboratory \\ Department of Electrical Engineering and Applied Physics \\ Case Westem Reserve University \\ Cleveland, Ohio 44106
}

\begin{abstract}
This paper describes a polygon micromotor scanner fabricated from single crystal silicon by deep reactive ion etching (DRIE) for in-plane scanning applications. The scanner consists of an outerrotor wobble micromotor with a $1.4 \mathrm{~mm}$-diameter, $200 \mu \mathrm{m}$-thick rotor. The outer-rotor design circumvents optical beam interference with wires or probes that provide electrical excitation to the motor. A variety of rotor polygon types were designed and fabricated, including those with 3, 4, 6, and 8 facets. After fabrication of the components, the scanners were assembled and operated for extended periods in room air. Typical scanners were operated at $60 \mathrm{~V}$. The optical beam was reflected off the sidewall of the polygon rotor using external optics at a wavelength of $1310 \mathrm{~nm}$. During dynamic optical measurements, $1.8 \mathrm{~mm}$ diameter gradient-index (GRIN) lenses were used as optical elements, and a repeatable signal was detected indicating less than $0.4^{\circ}$ of misalignment of the reflective surface due to rotor wobble or tilt.
\end{abstract}

\section{INTRODUCTION}

MEMS technology has been demonstrated for the application of optical scanning utilizing a variety of approaches. A $20 \mu \mathrm{m}$-tall reflective nickel polygon, plated on the surface of a polysilicon micromotor for in-plane scanning, was demonstrated in [1]. This device suffered large optical beam loss due to the small reflecting surfaces and required critical optical alignment. Optical scanning was demonstrated using a beam diffracted by grating elements that were etched into the relatively large-area rotors of polysilicon micromotors [2]. The device required out-of-plane optical alignment. Flip-up, vibrating, hinged mirrors that used resonant comb drive actuation for bar-code reading were demonstrated in [3]. The devices had fast scan speed, but limited scan angle and complex fabrication.

With the advent of DRIE technology, three-dimensional complex microstructures can be designed and fabricated with tall (100's of microns) geometries out of single crystal (SC) silicon, providing a unique flexibility in developing micromachined devices [4]. The absence of residual stresses in SC silicon imposes no limitations on the device's lateral dimensions. Integration with electronics is feasible, where the electronics can be fabricated anywhere on the surface, including the moving parts themselves. Optimized DRIE etch can potentially result in smooth sidewalls that may be suitable for optical applications. DRIE-fabricated, micromachined devices were reported recently including those for optical applications. Vertical, $75 \mu \mathrm{m}$-tall mirrors actuated by electrostatic comb drive for fiber-optic switching were reported in [5]. Devices with a $200 \mu \mathrm{m}$-tall mirror mounted on top of a stepping salient-pole micromotor to deflect an optical beam in the plane of the device to realize a 1 x8 optical switch were demonstrated in [6].

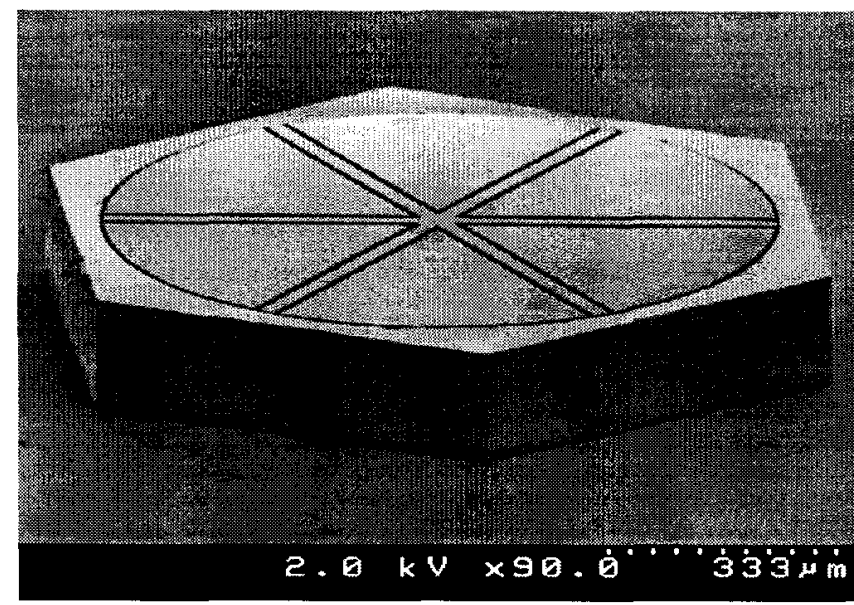

Figure 1. SEM photo of a typical DRIE fabricated polygon scanner using a hexagonal rotor. The rotor is $1.4 \mathrm{~mm}$ in diameter; and the reflecting sidewalls are $200 \mu \mathrm{m}$-tall.

\section{MICROSCANNER DESIGN}

The scanner micromotor design is based on the outer-rotor wobble motor in which the stator is folded inside the rotor, exposing the rotor outer radius sidewalls for optical coupling [7]. A means is required to prevent the rotor shorting to the stator during operation. The device presented here implements a hexagonal star-shaped bearing design between stator poles with protrusions that extend slightly beyond the stator (Fig. 1). These protrusions limit the rotor travel during its radial and angular motion to prevent it from shorting to the stator and define its points of role and slide (Fig. 2). The amount of bearing protrusion out of the stator determines the rotor/ stator gap during operation. The difference between the bearing radius and the rotor's inner radius, when the rotor is centered on the stator axis, determines the bearing clearance. Note that in operation, the rotor/stator gap is approximately $(\mathrm{g}$-d). The overall device

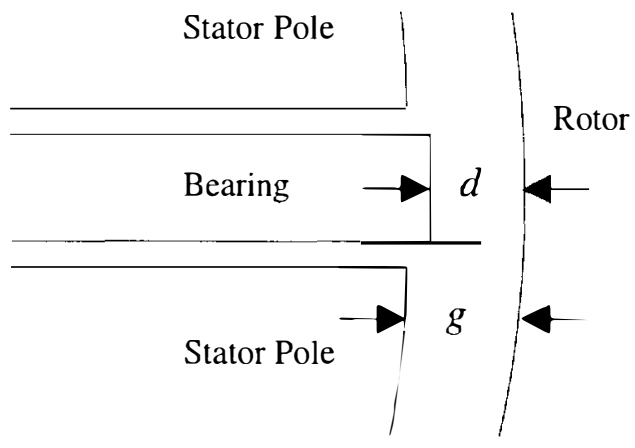

Figure 2. Top-view schematic of an outer-rotor wobble micromotor. With the rotor at the center of the stator, $d$ is the bearing clearance, and $g$ is the rotor/stator separation. 
dimensions were kept relatively large (1.4 mm-diameter, $200 \mu \mathrm{m}$ thick) to facilitate assembly and to provide large optical reflecting surfaces. These larger surfaces reduce optical beam losses, while relaxing optical alignment requirements. The outer rotor design also prevents optical beam interference with wires or probes that provide electrical excitation to the motor. Furthermore, in comparison to the micromotors reported in [6], rotor assembly to a corresponding stator is easier and was occasionally carried out manually without the need for a microscope or a probe station. The design resulted in relatively rugged motors, since it did not have fragile bearing posts at the center that are likely to break during assembly.

\section{MICROSCANNER FABRICATION}

The fabrication process is similar to that of the devices reported in [6] and will be described here briefly. Starting with a $200 \mu \mathrm{m}$-thick, silicon wafer that is fusion bonded to a $500 \mu \mathrm{m}$ thick, silicon substrate with $1.5 \mu \mathrm{m}$ of thermally-grown silicon dioxide, the recess pattern was defined and etched followed by the bushing definition and delineation in the rotor dies (Fig. 3). The rotor stator pattern was then defined and etchedusing DRIE through the top wafer, endpointing on the underlying oxide of the substrate. After a short phosphorous doping from a solid source, the wafer was diced into rotor and stator dies. The sacrificial oxide was stripped off the field area of the stator dies by a short BHF etch, while the rotor dies were fully released in $\mathrm{HF}$ and rinsed in DI water. Individual rotors were then collected and assembled onto corresponding stators. Rotors to be tested optically were Al-coated for improved reflectivity.

(a)

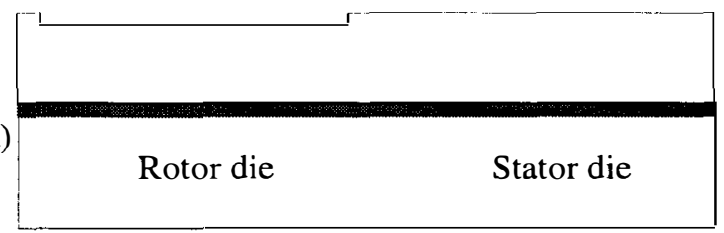

(b)

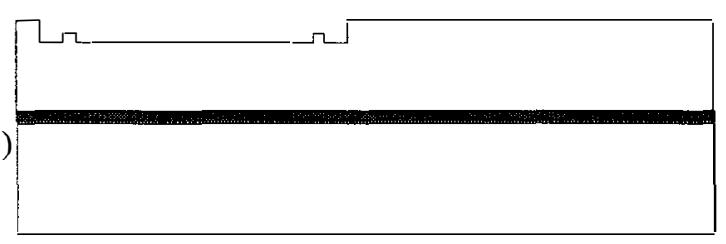

(c)

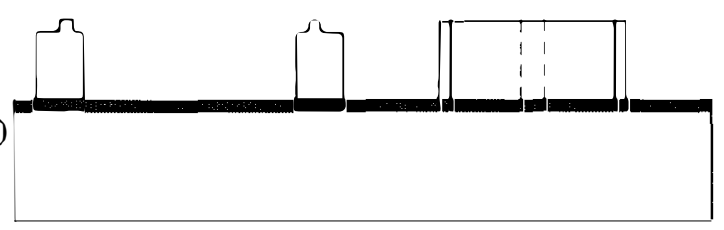

Silicon

Oxide (d)

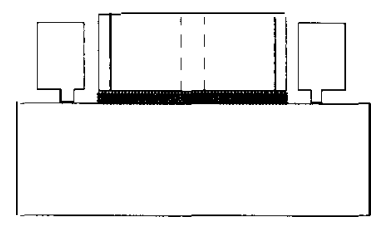

Figure 3. Cross-sectional schematics describing the micromotor fabrication process: (a) after recess definition and patterning to a depth of $8 \mu \mathrm{m} ;(b)$ after bushing definition and patterning to a depth of $2 \mu \mathrm{m}$; (c) after rotor/stator definition and DRIEpatterning through the top wafer; and (d) after rotor release and assembly onto the corresponding stator.

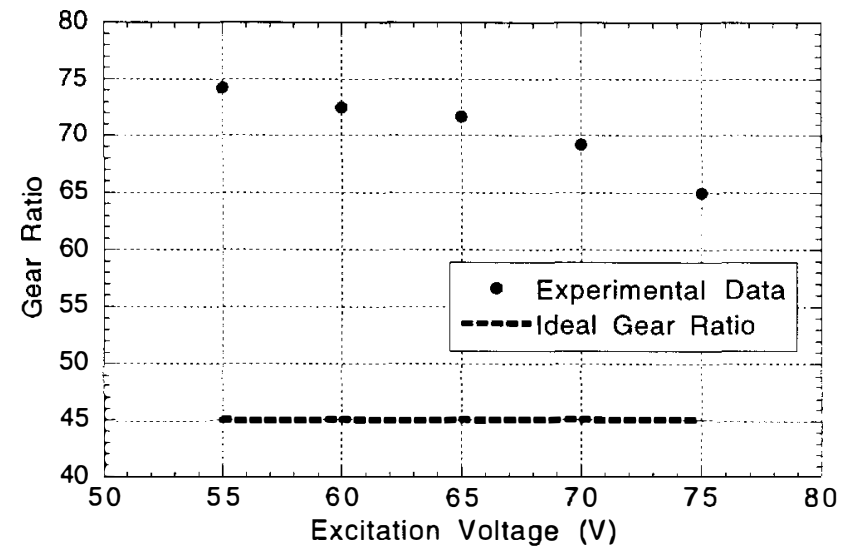

Figure 4. Gear ratio versus excitation voltage for the octagonal rotor micromotor. The $g$ and d parameters for this device are 13 and $11 \mu \mathrm{m}$, respectively. An excitation speed of $500 \mathrm{rpm}$ was used. The rotor gear ratio was measured by dividing the excitation speed by the average steady-state rotor speed.

\section{MECHANICAL MEASUREMENTS}

Upon assembling and testing the scanner actuation, it was verified that motor operation is highly DRIE etch dependent. The etch influences critical motor parameters such as rotor/stator gaps and bearing clearance. Great care is hence exercised to select rotor and stator patterns that are fully compatible given the initial design dimensions and their alteration due to the DRIE etch. It should be noted however that with a repeatable and reliable etch, these factors can be compensated for at the design stage. The inner rotor and bearing diameters of the device were $1005 \mu \mathrm{m}$ and $983 \mu \mathrm{m}$, respectively. The diameter of the stator was $979 \mu \mathrm{m}$. With an assembled device, the rotor/stator separation was $13 \mu \mathrm{m}$, and the bearing clearance was $11 \mu \mathrm{m}$. After release and assembly, the scanners were operated smoothly and reproducibly, even after storage in room air for extended periods (e.g., several months). The minimum operating voltage of the device was $50 \mathrm{~V}$. It was noticed that at high voltages (e.g., $>75 \mathrm{~V}$ ), the levitation force was strong enough to lift the rotor out of the plane of the substrate and result in poor device functionality. As a result, the operating voltage of the device was kept as low as possible, while maintaining the device operation. The micromotor gear ratio versus voltage is shown in Fig. 4. The higher experimental gear-ratio is due to the rotor slip, which increases with decreasing excitation voltage. Note that in this figure, the ideal gear ratio is defined as the bearing radius divided by the bearing clearance. Maximum operating speed when driven using a six-phase excitation to induce rotation was $58 \mathrm{rpm}$.

\section{OPTICAL MEASUREMENTS}

The scanner was tested dynamically using the experimental set up shown in Fig. 5. A 1310 nm laser source illuminated the scanner through a multi-mode fiber coupled to a gradient-index (GRIN) lens, which was positioned $10 \mathrm{~mm}$ from the rotor. The GRIN lens collimated the beam, preventing divergence and minimizing the beam diameter at the detector. Two germanium photo diode IR detectors, coupled to GRIN lenses through fiber, were placed in the plane of the scanner at fixed angles, separated by $30^{\circ}$, to receive the scanning beam. The GRIN lenses are highly sensitive to any angular misalignment in the beam, allowing the tilt in the device to be characterized. Electrical actuation of the device via the placement of probes, to contact the stator-poles, did not interfere with the optical 


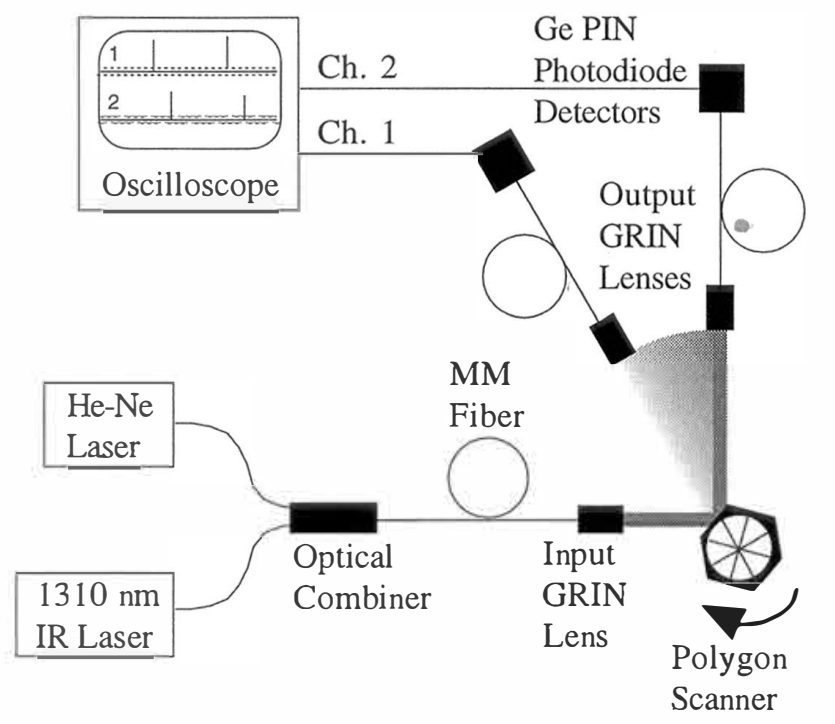

Figure 5. Schematic of the experimental setup used to perform optical testing on the scanner:

beam, since the stator is located inside the rotor.

For reflection off a given facet, the beam intensity remained high over large angles (Table 1). Of the light incident on the scanner, up to $-10.8 \mathrm{~dB}$ of optical power was observed at the detector. Much of the loss is due to the partial reflectivity of the $400 \mu \mathrm{m}$-diameter beam by the $200 \mu \mathrm{m}$-tall sidewall and due to the surface roughness of the sidewalls. To improve the coupling efficiency, a laser diode objective lens replaced the GRIN lens, with the spot focused near the scanner surface. The spot size of $125 \mu \mathrm{m}$ at the polygon surface was smaller than the height of the scanner surface, ensuring no losses due to partial beam reflection. The coupling efficiencies improved by about $5 \mathrm{~dB}$ and are shown in Table 1 . The spot size was also measured in the scanning plane, $2 \mathrm{~mm}$ after the facet. This yielded the scanner resolution, which was found to be $318 \mathrm{dpi}$.

The center point of the input beam was positioned off axis to a point slightly more than halfway to the outer radius of the stator. This produces a larger scan angle than an on-axis beam would. However, it results in beam translation, as well as angular motion during scanning. Using this arrangement, the beam angular scan range for the hexagonal rotor was seen to be $110^{\circ}$ with the parameters for the other facet numbers shown in Table 1. The detectors were placed within the scan arc and under electrical actuation. The beam was seen to scan repeatedly from detector 1 to 2 (Fig. 6) as the different facets reflected the beam.

Table 1. Experimentally determined relationship between number of facets and optical scanning parameters.

\begin{tabular}{|l|c|c|c|c|}
\hline Number of facets & $\mathbf{3}$ & $\mathbf{4}$ & $\mathbf{6}$ & $\mathbf{8}$ \\
\hline $\begin{array}{l}\text { Theoretical scan } \\
\text { angle }\end{array}$ & $120^{\circ}$ & $90^{\circ}$ & $60^{\circ}$ & $45^{\circ}$ \\
\hline $\begin{array}{l}\text { Scan line angular } \\
\text { range }\end{array}$ & $50^{\circ}$ & $80^{\circ}$ & $110^{\circ}$ & $95^{\circ}$ \\
\hline $\begin{array}{l}\text { Optical efficiency at } \\
\mathbf{9 0} \text { reflection using } \\
\text { GRIN lenses (dB) }\end{array}$ & -13.6 & -10.8 & -10.8 & -13.7 \\
\hline $\begin{array}{l}\text { Optical efficiency at } \\
\text { 90 reflection using } \\
\text { an objective lens (dB) }\end{array}$ & -9.2 & -6.3 & -5.6 & -6.6 \\
\hline
\end{tabular}

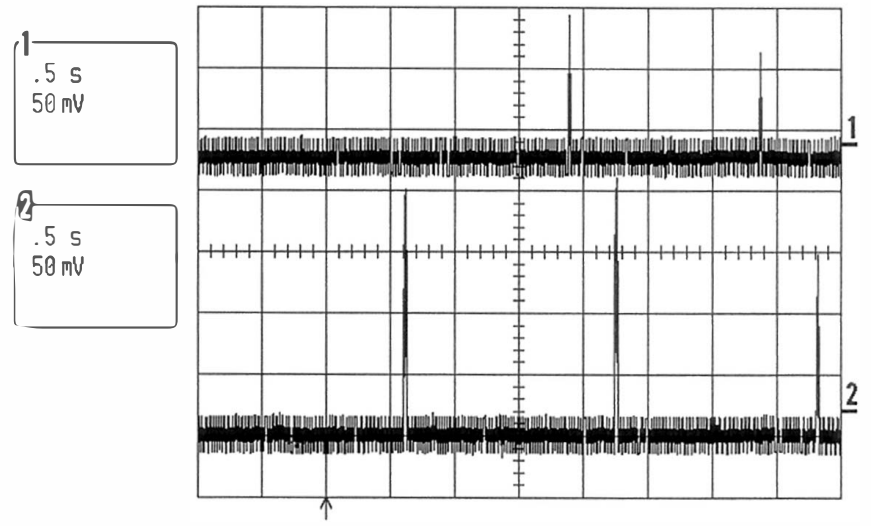

Figure 6. Oscilloscope trace of detected output optical power from the rotating hexagonal scanner. The detectors were at two fixed angles separated by $30^{\circ}$. Actuation signal speed was $500 \mathrm{rpm}$ at $55 \mathrm{~V}$.

\section{DISCUSSION}

The results in Table 1 indicate that more facets generally results in a larger scan angular range. Fewer facets yields better transmission of the optical beam over greater angles due to the larger lateral facet size. However, more facets yield a greater number of scans per second. A device with 6 or 8 facets would seem best at balancing these effects.

Even with the small spot size and Al-coated surface, the coupling efficiencies in Table 1 are still fairly low. In order to coat the polygons, they were mounted vertically on their side. The Alsputtering process only produced a uniform coating on exposed flat horizontal surfaces. Thus, any angled or vertical surfaces would have incomplete coating. For example, the top facet of a hexagon receives better coating than the adjacent two facets, and the remaining facets receive nearly no coating. The 3 -facet polygon had no horizontal facets exposed to the sputtering and had a relatively poor coating. Nearly all of this remaining loss is due principally to the sidewall roughness resulting from the DRIE etch. A close-up SEM photo of the scanner sidewall is shown in Fig. 7. The sidewall is relatively smooth over the top $40 \%$ of the surface (i.e., assembly results in a flipped rotor in Fig. 7) with an rms surface roughness, as reported in [5], of $360 \AA$. The roughness increases over the

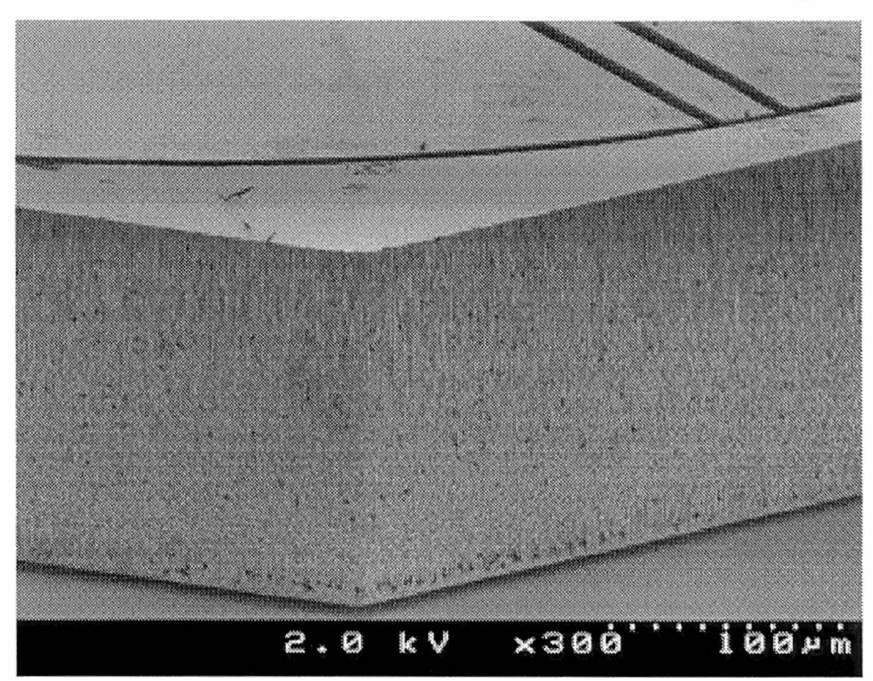

Figure 7. A close-up view of the etched polygon scanner sidewall from Fig. 1. Note that the rough area is on the top due to assembly. 
remainder of the sidewall surface until vertical grooves of nearly 1 $\mu \mathrm{m}$ in depth and width are observed near the base. The amount of feature undercut was as much as $10 \mu \mathrm{m}$. This limits the optical reflectivity by producing a large amount of scattering.

The scanning traces of Fig. 6 show the device operation from start-up to continuous rotation. Since the Ch. 1 and 2 GRIN lens pickups were placed at $60^{\circ}$ and $90^{\circ}$ from the input, respectively, they show different peak intensities due to the off axis position of the input beam. Spacing between the peaks is fairly consistent, indicating reliable motor operation. Measurement of the spacing on the trace yields a rotational speed of $6.76 \mathrm{rpm} \pm 0.09$. The mechanical measurements from Fig. 4 yield a rotational speed of $6.74 \mathrm{rpm} \pm$ 0.07 at $55 \mathrm{~V}$ for the same actuation speed. Since not only were different rotors used for the mechanical and optical measurements, but also different stators, these results indicate good repeatability of performance (and etch) for different devices.

The use of GRIN lenses for output beam detection allows the amount of angular variation in the rotor during actuation to be determined. For instance, a beam angle offset of $1^{\circ}$ corresponds to a loss of $12 \mathrm{~dB}$ for these GRIN lenses. In Fig. 6, the intensity varies by less than $35 \%$ giving a beam angle offset of less than $0.2^{\circ}$. Since the power readings were rarely less than $25 \%$ of the maximum value, this indicated a maximum angular offset of $0.8^{\circ}$ in the optical beam. Thus, despite the wobbling of the device as it rotated, it showed good repeatability of alignment with an out-of-plane tilt of the polygon of less than $0.4^{\circ}$.

The relatively low rotational speed of the device could be improved by two methods. The excitation speed or the bearing clearance could be increased to reduce the gear ratio. The latter option may create excessive wobbling, however, which would be detrimental to optical alignment.

To facilitate assembly, a decrease in feature size is desirable to create a greater clearance between the rotor and stator patterns. However, from a device perspective, large clearances are not desirable as they create excessive wobbling during operation. To address both of these issues, we envision a wafer scale assembly scheme in which a rotor wafer is placed on top of a stator wafer (similar to the flip-chip technique), hence speeding the assembly process and rendering the devices more suitable for batch fabrication. An additional issue with the scanner is that the rotor may disassemble if the device is turned upside down or is exposed to large mechanical shocks. This could be prevented if a cap is incorporated into the design (e.g., via an additional wafer bonding step) that extends slightly beyond the stator edge over the top of the rotor.

\section{CONCLUSIONS}

We have demonstrated an optical scanner based on a micromotor implementation using a DRIE fabrication approach. The large optical reflecting surfaces reduced optical power loss, while facilitating optical alignment. However, the roughness of the etched sidewalls contributed to optical loss. A repeatable signal was detected during device operations indicating a less than $0.4^{\circ}$ misalignment of the reflecting surface due to wobble or tilt.

\section{ACKNOWLEDGMENTS}

The authors would like to acknowledge STS Ltd., UK and AMMi Inc., Cleveland, OH for the DRIE etching, and R. Burla for help in the initial experiments. The work is supported in part by DARPA under Contract \#: DABT63-95-C-0070.

\section{REFERENCES}

1. K. Deng, H. Miyajima, V. Dhuler, M. Mehregany, S. Smith, F. Merat, and S. Furukawa, "The Development of Polysilicon Micromotors For Optical Scanning Applications," in Technical Digest, IEEE Solid-State Sensor and Actuator Workshop (Hilton Head, SC, June 1994), p. 234.

2. A. Yasseen, S. Smith, M. Mehregany, and F. Merat, "Diffraction Grating Scanners Using Polysilicon Micromotors," in Proceedings, IEEE Micro Electro Mechanical Systems Workshop (Amsterdam, Netherlands, Jan. 1995), p. 175.

3. M. Kiang, O. Solgaard, R. Muller, and K. Lau, "Surface Micromachined Electrostatic-Comb Driven Scanning Micromirrors for Barcode Scanners," in Proceedings, IEEE Micro Electro Mechanical Systems Workshop (San Diego, CA, Jan. 1996), p. 192.

4. E. Klaassen, K. Petrerson, J. Noworolski, J. Logan, N. Maluf, J. Brown, C. Storment, W. McCulley, and G Kovacs, "Silicon Fusion Bonding and Deep Reactive Ion Etching: A New Technology For Microstructures," in Sensors and Actuators A, Vol. 52, p. 132, 1996.

5. C. Marxer, C. Thio, M. Gretillat, N. de Rooij, R. Battig, O. Anthamatten, B. Valk, and P. Vogel, "Vertical Mirrors Fabricated by Deep Reactive Ion Etching for Fiber-Optic Switching Applications," in J. of Microelectromechanical Systems, vol. 6, no. 3, p. 277, September 1997.

6. A. Yasseen, J. Mitchell, T. Streit, J. Klemic, D. Smith, and M. Mehregany, "ARotary Electrostatic Micromotor 1x8 Optical Switch," in Proceedings, IEEE Micro Electro Mechanical Systems Workshop (Heidelberg, Germany, Jan. 1998), p. 116.

7. K. Deng, and M Mehregany, "Outer-rotor Polysilicon Wobble Micromotors," in Proceedings, IEEE Micro Electro Mechanical Systems Workshop (Oiso, Japan, Jan. 1994), p. 269.

8. Surface Technology Systems Ltd., Newport, UK, Available HTTP: http://www.stsystems.com. 